\title{
Método Híbrido para Avaliação de Jogos Baseado na Detecção Automática das Emoções dos Estudantes
}

\author{
Nelson N. Nascimento ${ }^{1}$, Francinete F. da Cunha ${ }^{1}$, Juliana C. Braga ${ }^{1}$, \\ João Paulo Gois ${ }^{1}$ \\ ${ }^{1}$ Universidade Federal do ABC (UFABC) \\ CEP 09210-580 - Santo André - SP - Brasil \\ \{nelson.junior, francinete.furtado, juliana.braga, joao.gois\}@ufabc.edu.br
}

\begin{abstract}
One of the approaches to evaluate the entertainment in educational games is to analyze their ability to provoke positive emotions in students. This evaluation is usually conducted through questionnaires applied before and after the match. This work proposes a method to evaluate educational games, based on the association of students' report data with data collected automatically from their emotional states during the gameplay. A literature review was performed to support the research and an experiment to validate the proposed method. The results indicate that recognizing students' emotional states during the game can support the development of motivating educational games.
\end{abstract}

Resumo. Uma das abordagens de avaliar diversão em jogos educacionais é analisar sua capacidade de provocar emoções positivas nos estudantes. Esta avaliação geralmente é realizada através de questionários aplicados antes e após a partida. $O$ objetivo deste estudo é propor um método para avaliar jogos educacionais, a partir da associação dos dados de relato pessoal dos estudantes com dados coletados automaticamente dos estados emocionais dos mesmos durante uma partida. Foi realizada uma revisão bibliográfica para embasar a pesquisa e um experimento para validar o método proposto. Os resultados indicam que reconhecer os estados emocionais dos estudantes durante o jogo pode apoiar o desenvolvimento de jogos educacionais motivantes.

\section{Introdução}

No cenário de um jogo educacional digital o designer cria o ambiente do jogo, mas não a experiência. Ela ocorre na medida em que o usuário joga. Analisar esta experiência, denominada Game User eXperience, é vital para a construção de jogos educacionais digitais envolventes. Pesquisas de Usuários de Jogos, em inglês Game User Research (GUR) [A. Drachen 2018], visam garantir uma experiência ideal para o usuário. Pesquisadores GUR aplicam métodos para avaliar o jogador, comunicando ao designer as reações do mesmo e quais recursos do jogo poderiam ser melhorados.

No contexto de um jogo educacional, os principais Métodos de Pesquisa de Usuários de Jogos para reconhecimento de emoções são: reconhecimento de emoções a partir de solicitação de informações ao usuário (por exemplo, questionários (autorrelato) [Pekrun et al. 2011], observadores externos [Truong et al. 2012] ou entrevistas [Bilal and Bachir 2007], entre outras formas); reconhecimento de emoções a partir do rastreamento de parâmetros implícitos (por exemplo, arquivos de $\log s$ 
[Kapoor et al. 2007], quantidade de erros ou tempo gasto para resolver um problema [Kardan and Einavypour 2008], quantidade de cliques do mouse [Lopatovska 2009] e dados de pressionamento de teclas [Hernandez et al. 2014], entre outros); reconhecimento de emoções a partir da fala (por exemplo, recursos extraídos do sinal de voz como a tonalidade, intensidade e velocidade da fala [Cao et al. 2015], entre outros); reconhecimento de emoções a partir de sinais vitais [Verma and Tiwary 2014] (aqui a emoção pode percebida através da visão (eletromagnética), do tato (mecânico, térmico), da audição (mecânico), do olfato e do paladar (químico) por meio de instrumentos como eletromiograma, eletrocardiograma, medidores de condutividade da pele, entre outros); reconhecimento de emoções a partir de gestos corporais (por exemplo, movimento do corpo [Darrell et al. 2000], movimento dos braços [Shimizu et al. 2007] e movimento das mãos e cabeça [Nickel and Stiefelhagen 2007]) e reconhecimento de emoções a partir de expressões faciais (ver Seção 3).

\subsection{Identificação do Problema}

De acordo com [Kiili et al. 2012], os procedimentos para criar um jogo educacional que seja capaz de promover uma aprendizagem eficaz e divertida, ainda permanecem pouco claros, principalmente em como manter os estudantes engajados em tarefas pedagógicas. Acreditamos que identificar automaticamente emoções dos estudantes enquanto experimentam um jogo educacional, combinadas com seus relatos pessoais (geralmente coletados em questionários aplicados antes e após o jogo), pode auxiliar a encontrar o equilíbrio entre motivação e aprendizagem no jogo.

\subsection{Objetivo}

Neste estudo propomos um método para avaliação do design de jogos educacionais, a partir da combinação dos dados de relato pessoal dos estudantes com informações coletadas automaticamente dos estados emocionais dos mesmos enquanto experimentam um jogo educacional.

\section{Trabalhos Relacionados}

[Eseryel et al. 2012] criaram um método para projetar e avaliar jogos MMORPGs (Massive Multi-Player Online Role-Playing Games), baseado em um modelo de interatividade entre o jogador, o ambiente e outros jogadores. Os autores pretendiam melhorar a motivação do aluno e suas habilidades de liderança, gestão e tomada de decisão. Estudantes da nona série de uma escola secundária rural no meio-oeste dos Estados Unidos participaram dos experimentos com o jogo McLarin's Adventures.

[Mysirlaki and Paraskeva 2010] propuseram um framework para design e avaliação de jogos educacionais para examinar os fatores pessoais e interpessoais envolvidos em um jogo multiplayer através da motivação intrínseca, engajamento e desempenho no jogo. Jogadores entre 12 e 16 anos foram selecionados aleatoriamente para responder questões sobre o jogo MMO, World of Warcraft. [Jovanovic et al. 2011] desenvolveram um metamodelo para projetar jogos educacionais, independente de plataforma, incorporando os princípios da teoria da motivação e interfaces multimodais. Os participantes tinham que construir um cenário para um jogo. A especificação do cenário tinha que ser detalhada, incluindo diálogos, descrições de cena, descrições de personagens, fluxo de comunicação, etc. 
[Jabbar and Felicia 2016] criaram um conjunto de melhores práticas baseadas no conceito de engajamento para projetar e implementar uma aprendizagem motivante baseada em jogos. Os estudantes participantes foram sorteados em uma escola nacional local e duas escolas no exterior e tinham entre 8 e 14 anos. [Elsattar 2017] propuseram uma metodologia de design e desenvolvimento de soluções de aprendizagem baseadas em jogos que facilitasse a integração de experiência de imersão com os elementos de jogo para apoiar a aprendizagem. Um jogo educacional digital foi criado para aumentar a conscientização para a geração Net sobre o uso excessivo da tecnologia em suas vidas sociais diárias.

Nosso trabalho compartilha semelhanças com os trabalhos anteriores, uma vez que, assim como os trabalhos relacionados, aplicaram seus modelos empiricamente e validaram seus experimentos em momentos pontuais através de questionários aplicados antes e após a execução das partidas. No entanto, nosso método se difere dos anteriores, pois, acrescenta uma avaliação contínua e automática da experiência do usuário, através do reconhecimento dos estados emocionais dos estudantes durante o jogo, combinando essas informações com os dados extraídos dos relatos pessoais dos estudantes nos questionários.

\section{Método}

O método proposto apoia o processo de avaliação de jogos educacionais, a partir das sensações experimentadas pelos estudantes durante uma partida. Ele combina informações sobre a experiência dos estudantes com o jogo, coletadas por dois instrumentos: (i) questionários aplicados antes e após a partida e (ii) algoritmo de reconhecimento de emoções pela análise das expressões faciais dos estudantes capturadas de maneira automática por uma webcam durante a partida.

Escolhemos o questionário pela sua simplicidade e por ser utilizado em praticamente todos os experimentos envolvendo avaliação da experiência do usuário em jogos educacionais. O método de reconhecimento da emoção através da face foi adotado por não necessitar de sensores específicos para identificação da emoção (bastando uma webcam), podendo ser replicado a um custo acessível e, por ser não invasivo (a webcam fica longe dos usuários, evitando desconfortos e não interferindo nos estados emocionais dos mesmos). A seguir um detalhamento sobre a metodologia utilizada para desenvolver cada um dos dois instrumentos.

\subsection{Questionário}

O método prevê a aplicação de dois questionários: pré-questionário, aplicado antes da interação com o jogo e pós-questionário, aplicado após interação com o jogo. Ambos podem ser elaborados de acordo com o que se deseja analisar no jogo educacional, ou seja, o método proposto nesse artigo, deixa esse aspecto em aberto. Na sessão 4, mostraremos a aplicação do método com um questionário específico.

\subsection{Algoritmo}

O algoritmo proposto possuiu como base teórica a Teoria Discreta da Emoção [Ekman et al. 1987] e o Sistema de Codificação da Ação Facial [Ekman et al. 1987]. Segundo essa Teoria, as emoções desencadeiam um padrão específico de respostas nos 
vários sistemas do corpo e são biológicas e consistentes entre as diferentes culturas. As emoções são classificadas em sete categorias: felicidade, tristeza, medo, raiva, nojo e surpresa mais o estado neutro. No Sistema de Codificação da Ação Facial cada emoção facial é resultado de contrações musculares temporárias dos elementos faciais, como nariz, olhos e região da boca (lábios) entre outros. A Figura 1 exibe as etapas do algoritmo de reconhecimento.

Figura 1. Etapas percorridas no Algoritmo.

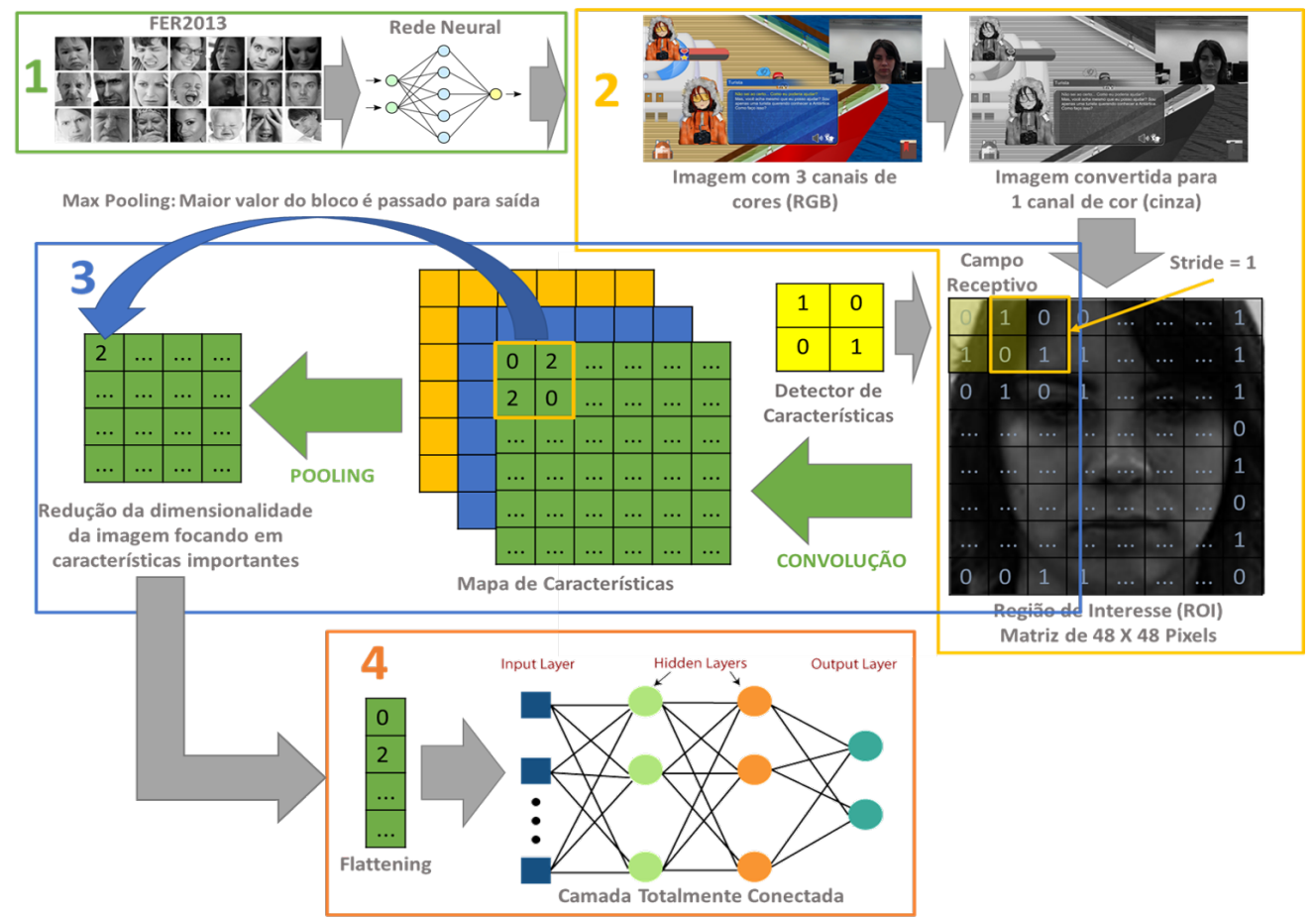

Fonte: Próprio Autor.

Na etapa 1 usamos para o treinamento da rede neural o banco de dados de expressões faciais FER-2013 (adotado em uma competição da Kaggle ${ }^{1}$ ) e a biblioteca Pandas $^{2}$ para a conexão com o banco. Na etapa 2, com a rede treinada, as imagens dos estudantes durante o jogo foram capturadas com 3 canais de cores (RGB) e, em seguida, convertidas em escalas de cinza. Após, as faces dos estudantes foram localizadas nessas imagens e atribuídas a uma região denominada "Região de Interesse" (ROI) tendo seus tamanhos normalizados em $48 \times 48$ pixels. Para o processamento das imagens foi utilizada a biblioteca OpenCV ${ }^{3}$.

Na etapa 3, com a região da face localizada e delimitada, as características dos componentes faciais (lábios, sobrancelhas, etc.) foram extraídas. Para esta etapa, e para a seguinte, foram utilizadas Redes Neurais Convolucionais através do emprego das bibliotecas TensorFlow $2.0^{4}$ e Keras ${ }^{5}$. A extração das características deu-se através de um

\footnotetext{
${ }^{1}$ https://www.kaggle.com/

${ }^{2}$ https://pandas.pydata.org/

${ }^{3}$ https://opencv.org

${ }^{4}$ Biblioteca de código aberto para aprendizado de máquina criado pelo Google Brain https://research.google/teams/brain/

${ }^{5}$ Biblioteca de rede neural de código aberto escrita em Python executada sobre o TensorFlow
} 
detector de características que percorreu cada porção da imagem da face (campo receptivo) em um passo $\mathrm{S}$ (Stride) em busca dos traços mais acentuados, produzindo um novo arranjo espacial e dimensional reduzido da imagem original (Mapa de Características). No exemplo, o $\mathrm{S}$ é 1, isto é, o detector deslocou-se pela imagem da face 1 coluna por vez. O Mapa de Características é gerado em dois momentos: (i) inicialmente por um processo chamado "Convolução", através do produto escalar entre a imagem da face e o Detector de Características e (ii) por um processo chamado "Pooling", que armazena o maior valor (Max Pooling) de cada bloco percorrido na imagem gerada na camada de convolução.

Por fim, na etapa 4, as imagens com as características faciais mais proeminentes que foram extraídas na etapa anterior e armazenadas em formato de matriz, foram convertidas para um formato de vetor (Flattening) e classificadas em uma das sete emoções (medo, raiva, nojo, felicidade, tristeza, surpresa e neutro) através do classificador prétreinado na etapa 1.

O método proposto engloba os dois instrumentos apresentados nessa seção inseridos em 4 etapas a serem detalhas na Seção 3.3, conforme ilustrado na Figura 2.

Figura 2. Etapas do Método Híbrido proposto.

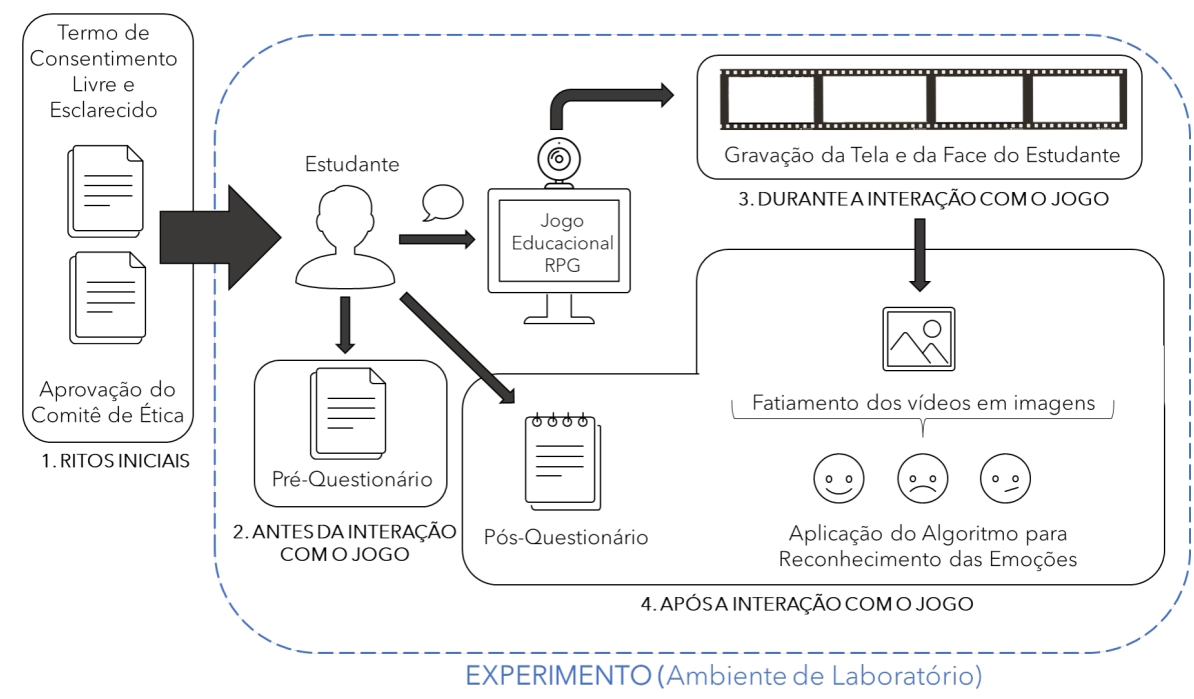

Fonte: Próprio Autor.

\subsection{Apresentação do Método Híbrido}

$\mathrm{Na}$ etapa 1 (Ritos Iniciais) deve ocorrer o preenchimento do Termo de Consentimento Livre e Esclarecido autorizando o uso das imagens e dos dados coletados para uso na pesquisa e a aprovação do Comitê de Ética. Nesta pesquisa o Termo de Consentimento encontra-se disponível em https://www.interaufabc.com.br/testes-do-jogo e a aprovação do Comitê de Ética deu-se sob o Parecer No 3.537.793. Na etapa 2 (Antes da interação com o jogo) deve ocorrer o preenchimento de um pré-questionário e o fornecimento de orientações básicas sobre como iniciar o jogo e sobre como será a condução do experimento. As instruções deste estudo podem ser acessadas em https://www.interaufabc.com.br/testes-do-jogo.

Na etapa 3 (Durante a Interação com o Jogo) cada estudante deve interagir com o jogo e as sessões precisam ser gravadas de forma que apareça tanto a tela do jogo quanto 
a imagem da face de cada participante. Na etapa 4 (Após interação com o jogo) cada estudante deve responder ao pós-questionário e, os vídeos gravados na etapa 3 , necessitam ser fatiados em imagens que representem os momentos de interesse no jogo (neste trabalho os momentos representam trechos do jogo que podem influenciar o engajamento) e que serão submetidas ao algoritmo para identificação de quais emoções emergirão nos estudantes durante a partida.

\section{Aplicação do Método}

Para aplicação do método proposto, escolhemos como objeto de estudo a missão Baleia do jogo educacional do gênero Role Play Game (RPG) denominado Antártica. O jogo foi desenvolvido pelos pesquisadores do grupo Intera ${ }^{6}$. No jogo, o jogador assume o papel de um expedicionário que sai do Ushuaia, de navio, a caminho da Península Antártica e realiza uma série de atividades que contribuem com pesquisas brasileiras de Citizen Science (Ciência Cidadã).

Aplicamos o método em novembro de 2019 em um laboratório de informática da Universidade Federal do ABC com computadores equipados com webcam e software ${ }^{7}$ para gravação simultânea da tela do jogo e da imagem da face do estudante. Ao todo, nove estudantes participaram do experimento. Destes, seis participantes tiveram seus dados considerados válidos (67\% dos alunos). Os dados de três estudantes não foram aproveitados, pois, as imagens de suas faces se encontravam fora do perímetro delimitado para a captação pela câmera ou continham algum tipo de oclusão (por exemplo, o queixo apoiado sobre a mão com os dedos obstruindo a região da boca).

Buscamos através da aplicação do método, compreender como as emoções de um estudante, enquanto experimentava o jogo, relacionavam-se com os elementos do jogo. Neste sentido, esperávamos ao final deste experimento responder a seguinte questão: "Qual a relação das emoções emergidas nos estudantes durante a sua interação com determinados momentos do jogo?". Para responder essa questão, aplicamos o algoritmo para identificarmos as intensidades das emoções que emergiam nas faces dos participantes durante a interação com o jogo, em nove momentos específicos. Geramos uma tabela com essas informações para os 6 alunos. No entanto, por uma limitação de espaço, a Figura 3 mostra um extrato dessa tabela apenas para o Aluno 4. Nela a segunda coluna, "Momento", representa os nove momentos que foram avaliados no jogo. A terceira coluna, "Emoção 1", representa a emoção com maior intensidade registrada em dado momento, para determinado aluno. A coluna quatro, “\% Emoção 1", representa a intensidade, em percentual, da emoção da coluna anterior. A quinta coluna, "Emoção 2", representa a próxima emoção com maior intensidade detectada para dado momento do jogo. A sexta coluna, “\% Emoção 2", representa a intensidade dessa segunda emoção em percentual. Da sétima até a décima terceira coluna são exibidas as seis emoções básicas mais o neutro e suas respectivas intensidades em percentuais em relação a cada um dos nove momentos escolhidos no jogo.

A partir da Tabela representada pelo seu extrato na Figura 3, geramos a Tabela 1 contendo a síntese das emoções que mais emergiram nos alunos ao se depararem com cada um dos nove momentos. Analisando a Tabela 1 concluímos que houve predominância das

\footnotetext{
${ }^{6}$ Grupo da UFABC que pesquisa o uso de tecnologias na educação - https://www.interaufabc.com.br/

${ }^{7}$ https://obsproject.com/pt-br/download
} 
Figura 3. Extrato para o Aluno 4, da Tabela que contêm a Relação dos 9 Momentos e as respectivas Intensidades das emoções dos Alunos.

\begin{tabular}{|c|c|c|c|c|c|c|c|c|c|c|c|c|}
\hline & Momento & Emoçāo 1 & \%Emocao 1 & Emoçāo 2 & \%Emoçāo 2 & \% Raiva & \% Nojo & \% Medo & \% Feliz & \% Triste & \% Surpreso & \% Neutro \\
\hline \multirow{9}{*}{ Aluno 4} & Acertar & Neutro & 35,39 & Triste & 25,1 & 16,3 & 0,74 & 16,31 & 3,65 & 25,1 & 2,52 & 35,39 \\
\hline & Associar Teia & Neutro & 46,88 & Triste & 21,34 & 12,21 & 0,26 & 14,31 & 2,68 & 21,34 & 2,32 & 46,88 \\
\hline & Conquistar Elementos & Triste & 28,01 & Raiva & 27,44 & 27,44 & 3,19 & 19,03 & 2,52 & 28,01 & 1,87 & 17,95 \\
\hline & Descobrir Pares & Neutro & 42,99 & Triste & 22,67 & 13,7 & 0,36 & 14 & 3,96 & 22,67 & 2,32 & 42,99 \\
\hline & Errar & Raiva & 35,17 & Triste & 22,9 & 35,17 & 3,02 & 15,82 & 2,06 & 22,9 & 1,14 & 19,9 \\
\hline & Fotografar Cauda & Triste & 37,29 & Neutro & 20,38 & 17,19 & 1,06 & 20,2 & 2,43 & 37,29 & 1,43 & 20,38 \\
\hline & Identificar Cauda & Triste & 34 & Medo & 22,56 & 16,71 & 1,33 & 22,56 & 3,07 & 34 & 2,77 & 19,56 \\
\hline & Interagir Mentores & Triste & 33,41 & Neutro & 24,4 & 15,44 & 1,07 & 20,4 & 2,8 & 33,41 & 2,48 & 24,4 \\
\hline & Ler Instruçōes & Raiva & 29,52 & Triste & 26,52 & 29,52 & 3,47 & 19,28 & 2,35 & 26,52 & 1,83 & 17,02 \\
\hline
\end{tabular}

Fonte: Próprio Autor.

emoções estado neutro, raiva e tristeza, em relação às emoções, felicidade, nojo, medo e surpresa.

Tabela 1. Síntese das Emoções que mais emergiram nos Estudantes.

\begin{tabular}{|c|c|c|c|c|}
\hline Aluno & \multicolumn{2}{|c|}{$\mathbf{1}^{\mathbf{a}}$ Emoção } & \multicolumn{2}{c|}{$\mathbf{2}^{\mathbf{a}}$ Emoção } \\
\hline Eetectada & $\begin{array}{c}\text { Quantidadade } \\
\text { de momentos } \\
\text { em que apareceu }\end{array}$ & $\begin{array}{c}\text { Emoção } \\
\text { Detectada }\end{array}$ & $\begin{array}{c}\text { Quantidadade } \\
\text { de momentos } \\
\text { em que apareceu }\end{array}$ \\
\hline Aluno 1 & Neutro & $8(89 \%)$ & Raiva & $1(11 \%)$ \\
\hline Aluno 2 & Neutro & $7(78 \%)$ & Tristeza & $2(22 \%)$ \\
\hline Aluno 3 & Neutro & $9(100 \%)$ & - & - \\
\hline Aluno 4 & Tristeza & $4(44 \%)$ & Neutro & $3(33 \%)$ \\
\hline Aluno 5 & Tristeza & $7(78 \%)$ & Neutro & $2(22 \%)$ \\
\hline Aluno 6 & Tristeza & $8(89 \%)$ & Neutro & $1(11 \%)$ \\
\hline
\end{tabular}

Também na aplicação do método, as palavras mais citadas nas respostas dos estudantes no pós-questionário, serviram como instrumentos de medição para ratificar os resultados obtidos no reconhecimento das emoções dos estudantes de maneira automática. Por exemplo, algumas questões do pós-questionário estavam diretamente relacionadas a fatores que poderiam levar o estudante a um estado de imersão no jogo, dentre os quais, objetivos claros, equilíbrio entre desafios propostos versus habilidades do estudante em realiza-los, feedbacks claros e imediatos, possibilidade real de aplicação e exibição do progresso do estudante.

A Tabela 2 exibe uma das questões do pós-questionário que, acreditamos, tem relação com o fator motivacional "feedback claro e imediato". Essa questão foi extrapolada para quatro momentos do jogo que demandavam alguma ação do estudante (associar teia, descobrir pares, identificar cauda e fotografar caudas), isso porque, nesses momentos, o estudante recebeu feedback por suas ações. A primeira coluna da Tabela 2 traz a pergunta e na segunda coluna são exibidas as respostas mais citadas pelos estudantes à essa questão.

\section{Análise do Re-Design do Jogo a partir do Método Proposto}

Com objetivo de avaliar o potencial de uso do método proposto, buscamos extrair algumas conclusões a respeito de qual a influência do design do jogo nas emoções dos estudantes a partir da associação das informações das emoções expressas nas faces dos estudantes 
Tabela 2. Respostas dadas à uma das Questões do Pós-Questionário relacionada com o Fator Motivacional "Feedback claro e imediato".

\begin{tabular}{|c|c|}
\hline $\begin{array}{l}\text { Perguntas do Pós-Questionario } \\
\text { relacionada ao fator motivacional } \\
\text { "Feedback Claro e Imediato" }\end{array}$ & $\begin{array}{l}\text { Respostas mais dadas pelos } \\
\text { Estudantes à estas Perguntas }\end{array}$ \\
\hline $\begin{array}{l}\text { O número de dicas está apropriado } \\
\text { dentro dos mini jogos? } \\
\text { Se não, indique os motivos }\end{array}$ & $\begin{array}{l}\text { - Sim: } 21 \% \\
\text { - Não. Fiquei desestimulado, por exemplo, porque } \\
\text { o painel lateral fora do campo de visão }(7 \%) \\
\text { - Não. As dicas deveriam ser mais esparsas e } \\
\text { contextualizadas (7\%) } \\
\text { - Não. O número de dicas foi insuficiente }(7 \%) \\
\text { - Não. As dicas eram superficiais }(7 \%)\end{array}$ \\
\hline
\end{tabular}

(algoritmo) com as informações sobre interação com o jogo (pós-questionário). Fazendo um recorte nos dados levantados, tendo como base o fator motivacional "feedback claro e imediato", produzimos algumas inferências. Ao analisarmos o pós-questionário, observamos pelas respostas dadas na Tabela 2 que as dicas e/ou feedbacks fornecidos pelo jogo estavam fora do campo de visão dos estudantes, fora de contexto, foram insuficientes e superficiais. Por outro lado, ao observarmos as emoções identificas automaticamente pelo algoritmo, verificamos que emoções positivas não emergiram, muito provavelmente pela "quebra" da imersão, neste caso, ocasionada por feedbacks mal projetados.

Concluímos que o método proposto ajudou a identificar elementos no design do jogo que se, mal projetados, podem provocar "quebras" no fluxo do jogo, ocasionando mudanças negativas no estado emocional dos estudantes. Da mesma forma, se bem elaborados, os componentes do jogo podem levar os estudantes a um estado imersão, fazendo emergir emoções positivas, aumentando o engajamento e a sensação de prazer dos mesmos ao jogar. Em resumo, acreditamos que o método proposto trouxe elementos para avaliar os jogos educacionais no sentido de deixá-los mais atraentes.

\section{Limitações}

A seguir, apresentamos algumas limitações importantes do experimento realizado e que podem ser utilizadas como aspectos a serem melhorados em trabalhos futuros: (i) A escolha de qual tela representaria cada um dos nove momentos selecionados para avaliar o jogo foi aleatória. Ao assistirmos as gravações dos vídeos, não observamos mudanças significativas nas expressões dos estudantes que justificasse considerar outros trechos. Cada estudante passava em média de 30 segundos a 1 minuto em determinada tela (momento) sem que sua expressão facial se alterasse. Portanto, qualquer imagem que fosse capturada, dentro desse trecho (intervalo de tempo), pressupomos, representaria a mesma emoção; (ii) Dos 9 participantes, 5 responderam o pré-teste e somente 1 por completo, comprometendo o levantamento demográfico da pesquisa; (iii) Das 9 gravações realizadas, 6 puderam ser aproveitadas. Os rostos de três estudantes estavam ocluídos ou fora do alcance da câmera. Além disso, os estudantes não foram filmados sob condições ideais de iluminação, o que pode ter comprometido o reconhecimento das emoções; (iv) Estados emocionais como ansiedade e frustração foram inferidos como sendo associados respectivamente a medo ou neutralidade e raiva ou tristeza e (v) Testamos nosso modelo com 
um jogo do tipo RPG (Role-Playing Game), necessitando ser testado com jogos de outros gêneros.

\section{Conclusões}

Acreditamos que esta pesquisa trouxe contribuições importantes sobre como avaliar jogos educacionais ao adicionar a este processo, geralmente realizado por questionários, o reconhecimento automático das emoções expressas nas faces dos estudantes enquanto jogam. Realizamos um experimento para correlacionar as emoções detectadas nas faces dos estudantes e as respostas (pós-questionário) sobre o que sentiram durante o jogo, com os elementos de design do jogo. Nele, emoções positivas como surpresa e felicidade praticamente não emergiram, de certa forma, pela maneira com que o jogo foi projetado. $\mathrm{O}$ jogo não foi considerado motivante pelos alunos e não contemplou certos aspectos, como "feedbacks claros e imediatos", o que contribuiu para a quebra do estado de imersão.

Como projeto futuro, sugerimos utilizar o mesmo algoritmo, porém com questionários de pré e pós teste relacionados ao conteúdo educacional para analisar se há alguma influência da emoção na aprendizagem. Estimulamos aplicar o modelo proposto em outros gêneros de jogos. Instigamos que sejam investigadas novas emoções, além das previstas no modelo teórico de Paul Ekman.

\section{Agradecimentos}

Realizamos o presente trabalho com apoio da Coordenação de Aperfeiçoamento de Pessoal de Nível Superior - Brasil (CAPES) - Código de Financiamento 001.

\section{Referências}

A. Drachen, P. Mirza-Babaei, L. N. (2018). Games User Research. Oxford Scholarship Online.

Bilal, D. and Bachir, I. (2007). Children's interaction with cross-cultural and multilingual digital libraries. ii. information seeking, success, and affective experience. Information Processing and Management, 43(1):65 - 80.

Cao, H., Verma, R., and Nenkova, A. (2015). Speaker-sensitive emotion recognition via ranking: Studies on acted and spontaneous speech. Computer Speech and Language, 29(1):186 - 202 .

Darrell, T., Gordon, G., Harville, M., and Woodfill, J. (2000). Integrated person tracking using stereo, color, and pattern detection. International Journal of Computer Vision, 37(2):175-185.

Ekman, P., Friesen, W., O’Sullivan, M., Chan, A., Diacoyanni-Tarlatzis, I., Heider, K., Krause, R., LeCompte, W., Pitcairn, T., and Ricci Bitti, P. (1987). Universals and cultural differences in the judgments of facial expressions of emotion. Journal of personality and social psychology, 53:712-7.

Elsattar, H. (2017). Designing for game-based learning model: The effective integration of flow experience and game elements to support learning. In 2017 14th International Conference on Computer Graphics, Imaging and Visualization (CGiV), pages 34-43, Los Alamitos, CA, USA. IEEE Computer Society. 
Eseryel, D., Guo, Y., and Law, V. (2012). Interactivity3 Design and Assessment Framework for Educational Games to Promote Motivation and Complex Problem-Solving Skills, pages 257-285.

Hernandez, J., Paredes, P., Roseway, A., and Czerwinski, M. (2014). Under pressure: sensing stress of computer users. In Proceedings of the SIGCHI conference on Human factors in computing systems, pages 51-60.

Jabbar, A. and Felicia, P. (2016). Towards a conceptual framework of gbl design for engagement and learning of curriculum-based content. International Journal of GameBased Learning, 6:87-108.

Jovanovic, M., Starčević, D., Minović, M., and Štavljanin, V. (2011). Motivation and multimodal interaction in model-driven educational game design. Systems, Man and Cybernetics, Part A: Systems and Humans, IEEE Transactions on, 41:817 - 824.

Kapoor, A., Burleson, W., and Picard, R. W. (2007). Automatic prediction of frustration. International Journal of Human-Computer Studies, 65(8):724 - 736.

Kardan, A. and Einavypour, Y. (2008). Involving learner's emotional behaviors in learning process as a temporary learner model. In Proceeding of International Conference on Virtual Learning (ICVL), Bucurest, Romania.

Kiili, K., de Freitas, S., Arnab, S., and Lainema, T. (2012). The design principles for flow experience in educational games. Procedia Computer Science, 15:78-91.

Lopatovska, I. (2009). Emotional aspects of the online information retrieval process. $\mathrm{PhD}$ thesis, Rutgers University-Graduate School-New Brunswick.

Mysirlaki, S. and Paraskeva, F. (2010). Intrinsic motivation and the sense of community in multiplayer games: An extended framework for educational game design. Informatics, Panhellenic Conference on, 0:223-227.

Nickel, K. and Stiefelhagen, R. (2007). Visual recognition of pointing gestures for humanrobot interaction. Image and Vision Computing, 25(12):1875-1884. The age of human computer interaction.

Pekrun, R., Goetz, T., Frenzel, A. C., Barchfeld, P., and Perry, R. P. (2011). Measuring emotions in students' learning and performance: The achievement emotions questionnaire (aeq). Contemporary Educational Psychology, 36(1):36-48. Students' Emotions and Academic Engagement.

Shimizu, M., Yoshizuka, T., and Miyamoto, H. (2007). A gesture recognition system using stereo vision and arm model fitting. International Congress Series, 1301:8992. Brain-Inspired IT III. Invited and selected papers of the 3rd International Conference on Brain-Inspired Information Technology "BrainIT 2006"held in Hibikino, Kitakyushu, Japan between 27 and 29 September 2006.

Truong, K. P., van Leeuwen, D. A., and de Jong, F. M. (2012). Speech-based recognition of self-reported and observed emotion in a dimensional space. Speech Communication, 54(9):1049 - 1063.

Verma, G. K. and Tiwary, U. S. (2014). Multimodal fusion framework: A multiresolution approach for emotion classification and recognition from physiological signals. NeuroImage, 102:162 - 172. Multimodal Data Fusion. 